\title{
Impact of work environment on cardiovascular diseases in Denmark
}

\author{
Ole Olsen, Tage S Kristensen
}

\begin{abstract}
Study objective-The aim was to estimate the quantitative impact of working conditions on cardiovascular diseases in Denmark.

Design-The study was based on a review of recent epidemiological research publications in which relative risks were estimated and risk factor prevalences were determined. The impact of working conditions was quantified by means of aetiological fractions.

Setting-The aetiological fractions were estimated on the Danish population.

Main results $-16 \%$ of the premature cardiovascular mortality in men and $22 \%$ in occupationally active women is avoidable through interventions in the work environment. If "sedentary" work is included in the occupational risk factors, the aetiological fractions reach $51 \%$ for men and $55 \%$ for women. Taken separately, the major aetiological fractions for cardiovascular risk factors at work are respectively (women in parentheses) $6 \%$ (14\%) for monotonous high paced work, $7 \%$ (7\%) for shift work, and $2 \%(2 \%)$ for passive smoking.

Conclusions-The aetiological fractions show that working conditions play a considerable role in cardiovascular diseases. Furthermore they might widen the focus of preventive cardiology from interventions directed only at individual and lifestyle risk factors to interventions directed also at working conditions.
\end{abstract}

The epidemiology of cardiovascular disease has for many years focused on the significance of the conventional individual risk factors for the incidence of cardiovascular diseases, while factors in the social and occupational environment have attracted less interest. Looking at occupational medicine we find a similar picture: much research has been performed on work and cancer, reproductive failures, musculoskeletal diseases etc, but little attention has been paid to cardiovascular diseases.

In 1989 two systematic and extensive reviews of the epidemiologic literature on work environment exposures and cardiovascular diseases were published. ${ }^{12}$ In these reviews it was concluded that the research on work environment and cardiovascular diseases-although at many points still insufficient and of unsatisfactory qualityhas today reached a level that makes it possible to identify a number of risk factors in the work environment with reasonable certainty. An occupational risk factor for cardiovascular diseases is in this article defined as any factor in the work environment that is causally related to cardiovascular diseases, that is any factor that directly or through one of the conventional risk factors (such as blood pressure) increases the risk of getting a cardiovascular disease.

The aim of the present paper is to some extent similar to the aim of the paper by Fox and Adelstein: "Occupational mortality: work or way of life?". ${ }^{3}$ But the calculations in the present paper are-though still crude-based on more explicit criteria. The purpose of the present paper is to estimate the relative impact of the work environment on the incidence of premature cardiovascular disease. This is done by estimating the aetiological fractions of the relevant work environment factors for men and women in Denmark. By substituting the estimates used for the different exposures with estimates for other countries the results can be applied to these countries as well. The aetiological fractions can be considered as estimates of the potential benefit to be gained by eliminating a given occupational risk factor. This measure is therefore essential in formulating a rational strategy for the prevention of cardiovascular disease.

\section{Methods}

MATERIAL

Two kinds of information are needed to quantify the influence of the work environment on premature cardiovascular diseases in a particular country: (1) the risk associated with each risk factor; and (2) the prevalence of each risk factor. In the following section the basis on which the risk is quantified in terms of relative risk is described. The subsequent section contains an assessment of the prevalence of the risk factors.

\section{Origin of the applied relative risks}

In the above mentioned reviews by Kristensen ${ }^{12}$ the methodological quality of the epidemiological studies on work environment factors and cardiovascular disease was evaluated using five criteria: (1) control with the time dimension; (2) control with confounding variables; (3) control with selection processes; (4) quality of measurements of exposures and diseases; (5) quality of design and statistical analysis. On the basis of this evaluation each study was given a score between " $x$ " (very poor) and "xxxxx" (extremely good). The next step was to evaluate the overall evidence (for causality) concerning each potential risk factor. Here three criteria were used: (1) the number of studies; (2) the quality of the studies (with more weight given to the best 
studies); (3) the consistency of the study results. This two step evaluation resulted in the classification of occupational risk factors shown in table I. Here quantifications will be made for the risk factors in the two groups: "Very definite" and "Quite definite". The following approach does not assume consensus regarding the causal relationship between each work environment factor and cardiovascular diseases. By presenting the assumptions and calculations explicitly, it is then possible for readers who disagree with the categorisation of the relationships used here to conduct their own calculations.

Table I Classification of possible cardiovascular risk factors in the work environment.

\begin{tabular}{|c|c|c|}
\hline \multirow[b]{2}{*}{$\begin{array}{l}\text { Causal relation to } \\
\text { cardiovascular diseases }\end{array}$} & \multicolumn{2}{|l|}{ Type of risk factor } \\
\hline & Non-chemical & Chemical \\
\hline Very definite & Physical inactivity at work & $\begin{array}{l}\text { Carbon disulphide } \\
\text { Nitroglycerin/nitroglycol }\end{array}$ \\
\hline Quite definite & $\begin{array}{l}\text { Work strain (high demands } \\
\text { and low influence) } \\
\text { Shift work } \\
\text { Noise }^{\text {a }}\end{array}$ & $\begin{array}{l}\text { Lead }^{\mathrm{a}} \\
\text { Passive smoking }\end{array}$ \\
\hline Quite possible & & $\begin{array}{l}\text { Cobalt } \\
\text { Arsenic } \\
\text { Combustion products }\end{array}$ \\
\hline Possible & $\begin{array}{l}\text { Heat }{ }^{\mathrm{b}} \\
\text { Irradiation } \\
\text { Power frequency magnetic } \\
\text { fields } \\
\text { Low frequency noise }\end{array}$ & $\begin{array}{l}\text { Organophosphates } \\
\text { Dinitrotoluene } \\
\text { Antimony } \\
\text { Beryllium } \\
\text { Cabon monoxide }^{\mathrm{b}}\end{array}$ \\
\hline Probably no relationship & $\begin{array}{l}\text { Microwaves } \\
\text { Cold }^{\text {b }}\end{array}$ & $\begin{array}{l}\text { Cadmium } \\
\text { Organic solvents }{ }^{c}\end{array}$ \\
\hline
\end{tabular}

When (or if) the causal relationships are accepted the next step is to quantify the strengths of these relationships. This has to some extent been done by Kristensen ${ }^{12}$ by means of relative risks. Of course this is a rough and simplistic measure: the relative risks might depend on age, on other risk factors, on the specific dose, etc. But it has been judged to be an adequate simplification in order to reach a first quantification, which can be used in the discussion of different preventive strategies. The relative risks proposed in ${ }^{12}$ are based on a thorough evaluation of the epidemiological quality of the studies and an educated "weighted" average of the relative risks in the individual studies taking into accoun methodological strengths and weaknesses, biological plausibility, consistency among studies, etc. The influence of occupational risk factors can operate either directly on the incidence of cardiovascular diseases or through intermediate individual risk factors. The individual risk factors, blood pressure and serum cholesterol, were regarded as being causal risk factors for cardiovascular diseases. So was smoking, but as both starting smoking and stopping smoking are determined by many other social and cultural factors than working conditions, smoking was not considered a mediator between work environment and cardiovascular diseases. Consequently an occupational factor is considered a risk factor for cardiovascular diseases if it either raises the blood pressure or the level of serum cholesterol, or directly raises the incidence of cardiovascular diseases. The relative risks are presented in table II. The relative risks for work strain and for noise are not explicitly stated in reference. ${ }^{1}$ The estimates of the former vary between 1.3 and about 4 in different studies, with a slight tendency for higher values to be found in the better studies. Here the relative risk is fixed at 2 . The quality of the epidemiological studies concerned with noise is relatively lower and the bias is mostly towards the null hypothesis. The causal relationship between noise and cardiovascular diseases seems to be through an increase in blood pressure of 5 to $15 \mathrm{~mm} \mathrm{Hg}$ among those exposed. A reasonable estimate might be a relative risk of $1 \cdot 2$, which is used here.

As mentioned above, the main interest is in premature cardiovascular disease. But cardiovascular diseases have many manifestations and are categorised in many different ways and subgroups: angina pectoris, ischaemic heart disease (lethal or non-lethal), stroke, etc. The relative risks and therefore the calculations based on them have to be interpreted as relevant to a broad perception of cardiovascular diseases. We have chosen-or to some extent been forced-to do this because the studies reviewed ${ }^{12}$ are not consistent with regard to end points. The choice is to some extent supported by Kannel, ${ }^{4}$ p 282: "The major atherosclerotic diseases share most precursers and a common pathogenesis ... These facts strongly suggest that prophylactic measures designed to prevent one may prevent the others as well". With regard to early mortality (below the age of 70), which is of particular concern, most cardiovascular disease is ischaemic heart disease. In Denmark this proportion is $75^{\circ}$ o.

The prevalence of the occupational risk factors Besides the relative risks the prevalence figures of the occupational risk factors are needed to quantify the impact on cardiovascular diseases. Contrary to expectations the task of obtaining these figures turned out to be a difficult one. The chemical exposures of interest here are rare in Denmark (with lead being perhaps the only exception) and an inquiry to the Danish National Institute of Occupational Health and the Danish Labour Inspection Service revealed that the data concerning the prevalence of these chemical exposures are quite poor. These risk factors have therefore been omitted in our calculations. The estimated prevalences of the rest of the occupational risk factors have been obtained in various ways, which will be described below.

The population of interest is the occupationally active part of the population which consists of 1.5 million men aged $15-74$ and 1.25 million women aged 15-74. Overall within this age range, there are 1.9 million of each sex. The figures presented below are prevalence figures, ie, they indicate how many people are being exposed at a given point in time. But mobility in the labour market means that substantially larger numbers have been exposed at some time in their lives.

Monotonous high paced work-The prevalence of monotonous high paced work has been estimated according to the numbers employed in the relevant job categories, such as drivers: 55 000; waiters: 5000; etc. In total these figures sum to 110000 men. However not everyone in 
these categories necessarily has monotonous high paced work, so the figure is reduced to 90000 , which is equivalent to $6 \%$ of occupationally active males. Among women, high paced work is more widespread. An estimation similar to that for men leads to an estimated prevalence among occupationaly active women of $16 \%$. Compared to the definition of monotonous high paced work in the research referred to in $^{1}$, the above enumeration is based on a more restrictive definition.

Working hours-In a representative survey of the Danish population aged 20-69 years conducted by the Danish Institute of Social Research in $1976,{ }^{5} 5000$ people were asked about their working hours. This showed that the prevalence of shift work and other unsocial working hours was approximately $20 \%$ for both sexes.

Noise-Regarding noise, no good surveys have been performed in Denmark. Based on Dutch and Norwegian investigations, the Danish Labour Inspection Service estimates that 150000 persons are exposed to sound levels above $90 \mathrm{~dB}$. This is a very imprecise estimate. Despite the inappropriateness of measuring sound instead of noise as a risk factor for cardiovascular diseases, ${ }^{1}$ much research has used a limit of $90 \mathrm{~dB}$. As more men than women are exposed to noise, it has been decided to use the figures of 100000 men and 50000 women equivalent to $7 \%$ and $4 \%$ respectively exposed to noise among the working population.

Passive smoking-No surveys regarding passive smoking at the workplace existed prior to the present investigation. Through the Danish National Institute of Social Research, a survey was performed in $1987,612 \%$ of working men were exposed to passive smoking and $13 \%$ of the women. Passive smokers include only nonsmokers; the percentage is of all persons (including smokers). To be classified as passive smokers, the non-smokers had to be exposed the whole day or part of the day and not just at meetings of during breaks.

Physical activity-Physical activity has to be both vigorous ${ }^{78}$ and dynamic to reduce the risk of cardiovascular diseases. Static muscle work does not have any beneficial influence on the risk of cardiovascular diseases. As society has changed, it is hard to find any job categories with sufficiently dynamic muscle work. Most work is sedentary or static muscle work: office work, transportation, surveillance, etc. Postmen and ballet dancers are among the few with sufficient dynamic muscle work. We have estimated that at most $10 \%$ of the working force have enough dynamic, aerobic muscle work during working hours. In other words, $90 \%$ of the working population is judged to have "sedentary" work - the quotation marks indicating that sedentary work is defined to include static muscle work. As people with high physical activity during leisure time will have only a minor beneficial effect from physical activity during working hours, figures showing the prevalence and intensity of physical activity in leisure time are needed. In a recent survey ${ }^{9}$ the Danish Institute of Clinical Epidemiology has estimated that approximately $20 \%$ of the occupationally active population with "sedentary work" indulge in sports, digging the garden or the like, for at least four hours a week.

\section{PROCEDURE}

The impact of working conditions on public health is expressed by means of the aetiological fraction. The concept was introduced by Levin in $1953,{ }^{10}$ the term "etiological fraction" and the abbreviation, "EF", by Miettinen in 1974. ${ }^{11}$ The concept has sometimes ${ }^{12}$ been defined by the following simplistic formula: (RR-1) $\star b /[(R R-$ $1) \star b+1]$, where $R R$ is the relative risk and $b$ is the fraction exposed. This is, however, in many circumstances inappropriate. ${ }^{13}$ It is far more correct to state a verbal definition, as done by several authors. ${ }^{1011} 14$ The phrasings are somewhat different but the meaning is the same as that of the following definition:

The aetiological fraction is a concept defined for a specific disease or group of diseases (here: cardiovascular diseases) in a given population (here: the Danish) and has the following theoretical meaning: "The aetiological fraction is that proportion of the disease that would not have occurred had the risk factor not occurred in the population."

If a certain factor is not a causal agent, but only a risk indicator, the aetiological fraction is zero, as removal of the factor would not change the disease incidence. Because it is impossible to reach absolute certainty as to whether a factor has a causal relationship to a disease or not, we can never be certain that the numerical calculation of an aetiological fraction is correct. However, by agreeing upon causality and the strength of the causal associations as well as the distribution of risk factors in the population, it is possible to obtain an estimate of the aetiological fraction. As the definition of the aetiological fraction is made in the context of a hypothetical situation ("if the risk factor had not occurred"), caution is needed in the interpretation of the numerical calculation and in the translation into preventive potential. If everybody stopped smoking today neither the prevalence nor the incidence of cardiovascular diseases would drop immediately to the level found in persons who have never smoked.

Ideally we ought to know both the simultaneous distribution of all risk factors in the population and the risk associated with each combination of risk factors appearing in the population in order to calculate the aetiological fraction. This is not the case, so we shall have to make some assumptions.

All the occupational risk factors are assumed to be simultaneously independent. Furthermore the occupational risk factors and the conventional risk factors which are not intermediate are also assumed to be independent. The only exception is active and passive smoking, since passive smoking is defined in such a way that it occurs only among non-smokers.

The strength of associations has already been reported by means of one relative risk for each risk factor. This implies that a multiplicative model is assumed, or in other words that, eg, the relative risk of working in noise is the same irrespective of whether the work is "sedentary" or not, shift work or not, etc. or whether the exposed person is a smoker, a vegetarian, etc. The reported relative 
risks have to be interpreted as adjusted relative risks which means that, in the "educated averaging" of the estimated relative risks, most emphasis has been put on studies with the best control for confounding.

As the occupational risk factors in general have not been recognized, the studies of one occupational risk factor seldom consider other occupational factors explicitly. But in a way they are implicitly kept under control because the studies are either randomized or restricted in such a way that the occupational risk factors not under consideration are often the same in the exposed (index) and the unexposed (reference) group.

With the assumptions made it is possible to use the simplistic formula to estimate the aetiological fractions. Two exceptions have to be made regarding the straightforward application of the formula. When the aetiological fraction for "sedentary" work is estimated, the value of b has to be defined somewhat differently, because those persons who are physically active in their leisure time are assumed to gain no positive effect of further exercise. So b is set at $72^{\circ}{ }_{0}$, namely the $90^{\circ}{ }_{0}$ with "sedentary" work minus $18^{\circ}{ }_{0}\left(20^{\circ}{ }_{0}\right.$ of $90^{\circ}{ }_{0}$ ) having "sedentary" work but being physically active in their leisure time. The aetiological fraction for passive smoking is calculated according to the following expression:

$\left(R R_{p}-1\right) \star b_{p} /\left[b_{a} \star R R_{a}+b_{p} \star R R_{p}+\left(1-b_{p}-b_{a}\right)\right]$ where $R R_{p}$ and $R R_{a}$ are the relative risks for passive and active smoking respectively, and $b_{p}$ and $b_{a}$ are the fractions of passive and active smokers. The values $R_{\mathrm{a}}=2.5$ and $b_{a}=50^{\circ}{ }_{0}$ are used in the calculations for both sexes.

Additionally aetiological fractions for "all working conditions" are reported. These require a minor extension of the definition of aetiological fractions to handle the fraction of a set of risk factors and not just of a single risk factor. ${ }^{14}$ The definition then reads: "The aetiological fraction is that proportion of the disease that would not have occurred, had the set of risk factors not occurred in the population". With the assumptions made about the mutually independent distribution of the occupational risk factors and their multiplicative effect, it is possible to calculate the aetiological fraction for a set of risk factors (here called $\mathrm{a}, \mathrm{b}, \ldots, \mathrm{n}$ ) according to the following formula: ${ }^{10}$

$$
\mathrm{EF}(\mathrm{all})=1-[1-\mathrm{EF}(\mathrm{a})][1-\mathrm{EF}(\mathrm{b})] \ldots[1-\mathrm{EF}(\mathrm{n})]
$$

Note that the aetiological fraction for the set of risk factors is not the sum of all the aetiological

Table II Aetiological fractions of work environment risk factors for premature cardiovascular diseases in Denmark.

\begin{tabular}{|c|c|c|c|c|c|}
\hline \multirow[b]{2}{*}{ Risk factor } & \multicolumn{2}{|c|}{$\begin{array}{l}\text { Prevalence of } \\
\text { exposures }\left(\begin{array}{c}0 \\
0\end{array}\right)\end{array}$} & \multirow{2}{*}{$\begin{array}{l}\text { Relative } \\
\text { risks }\end{array}$} & \multicolumn{2}{|c|}{$\begin{array}{l}\text { Aetiological } \\
\text { fractions }\left({ }^{\circ},\right)\end{array}$} \\
\hline & Men & Women & & Men & Women \\
\hline $\begin{array}{l}\text { Monotonous, high paced work } \\
\text { Shift work, etc } \\
\text { Noise } \\
\text { Chemical exposures } \\
\text { Passive smoking } \\
\text { "Sedentary" work }\end{array}$ & $\begin{array}{c}6 \\
20 \\
7 \\
\text { low } \\
12 \\
90^{\mathrm{a}}\end{array}$ & $\begin{array}{l}16 \\
20 \\
4 \\
\text { low } \\
13 \\
90^{\mathrm{a}}\end{array}$ & $\begin{array}{r}2 \cdot 0 \\
1 \cdot 4 \\
1 \cdot 2 \\
>1 \cdot 0 \\
1 \cdot 3 \\
2 \cdot 0\end{array}$ & $\begin{array}{c}6 \\
7 \\
1 \\
0-1 \\
2 \\
42\end{array}$ & $\begin{array}{r}14 \\
7 \\
1 \\
0 \\
2 \\
42\end{array}$ \\
\hline \multicolumn{3}{|c|}{$\begin{array}{l}\text { All occupational risk factors } \\
\text { All occupational risk factors except "sedentary" work }\end{array}$} & & $\begin{array}{l}51 \\
16\end{array}$ & $\begin{array}{l}55 \\
22\end{array}$ \\
\hline
\end{tabular}

But only those $72^{\circ}$, who are also
from physical activity at work. fractions involved. Nor is there any reason for expecting this ${ }^{15} 16$ as many events can be prevented in more than one way, but never more than once.

\section{Results}

The relative risks, the prevalence of exposures, and the calculated aetiological fractions for each risk factor are presented in table II. Additionally two proposals for the aetiological fraction for "all working conditions" are reported. The first of these is calculated for the set of all occupational risk factors. The second one is calculated for the same set, excluding "sedentary" work. The reason for calculating the second aetiological fraction is the possibility of compensating for "sedentary" work by being physically active in leisure time. It is not possible in the same way to avoid the increased risk associated with the other working conditions in leisure time.

As already mentioned the disease entity "cardiovascular diseases" is only loosely defined in the above estimations. Therefore the reported aetiological fractions can be translated into absolute figures in different ways, depending on the purpose, eg, number of first myocardial infarctions, number of disability pensions, etc. Morbidity is relevant when quantifying loss in quality of life or burden on the health care system, but morbidity is not very well registered. Mortality is described in death registers and might serve as an example of the calculation of preventive potential.

In Denmark approximately 4200 males and 1700 females die from cardiovascular diseases each year before age 70 . Assuming that all the male deaths and $70^{\circ}{ }_{0}$ of the female deaths occur among persons who have been occupationally active, this means that $2100\left(51^{\circ}{ }_{0}\right.$ of 4200$)$ premature deaths among males and $700\left(55^{\circ}{ }_{0}\right.$ of $70^{\circ}{ }_{0}$ of 1700 ) deaths among females could have been avoided if none of the occupational risk factors had occurred. If we disregard "sedentary" work, the numbers would be 700 males and 300 females per year. With an age limit of 60 the latter numbers would be 200 and 100 .

\section{Discussion}

To our knowledge this is the first time the impact of the work environment on the incidence of cardiovascular diseases has been estimated by means of an aggregate aetiological fraction, although the question has been discussed previously. ${ }^{3}$ Aetiological fractions for some of the specific occupational risk factors have been calculated for populations similar to the Danish. In Swedish calculations based on different follow up studies ${ }^{17}$ monotonous high paced work had aetiological fractions in the range $3-7^{\circ}{ }_{0}$ for men and $9-13^{\circ}{ }_{0}$ for women. These are comparable to our figures. For lower age limits the estimate for men was larger. In a study from New Zealand ${ }^{18}$ passive smoking in the work place had aetiological fractions of $10^{\circ}{ }_{0}$ in men and $7^{\circ}{ }_{0}$ in women. This is larger than our findings, and mostly due to application of a larger relative risk based on the assumption that exposure to passive smoking in the work place is of greater intensity than 
exposure to spousal smoking. Without this assumption the estimates would be in closer agreement with ours. Aetiological fractions for "sedentary work" specifically have not been calculated elsewhere, but figures for physical inactivity in general in the US population ${ }^{19}$ lead to aetiological fractions in the same order of magnitude as here.

For comparison, some aetiological fractions calculated for non-occupational risk factors are listed below without further discussion. The estimates are based on samples which are not representative for the countries, but relate respectively to middle aged Swedish men from Gothenborg $^{20}$ and US college alumi ${ }^{21}$ : hypertension: $17 \%$ and $9 \%$; smoking: $39 \%$ and $25 \%$; parental history: $12 \%$ and $11 \%$; serum cholesterol (in the Swedish study only): $38 \%$. Both sets of authors note that the sum of aetiological fractions may exceed $100 \%$. This is in fact the case in the Swedish study, but not in the American.

Theoretical papers ${ }^{15} 16$ also stress that the sum of the aetiological fractions may exceed $100 \%$. There are three important interpretations of this. First, the conceptual interpretation is that even though the aetiological fractions for some risk factors are large, this does not exclude the possibility that other risk factors may also have a large influence on disease incidence. In other words the fact that occupational risk factors have a large impact on cardiovascular diseases does not contradict the fact that the traditional risk factors (smoking, cholesterol, etc) have a large impact. Second, the preventive interpretation is that it is possible to choose between completely different preventive strategies to obtain similar decreases in disease incidence, when the sum of aetiological fractions is large, ie, interventions directed only at smoking, only at the work environment, etc. Third, the scientific interpretation is that even though the sum has exceeded $100 \%$, the research ought not to stop. It is still possible that more risk factors might be identified, and that these could lead to cheaper or more convenient preventive alternatives.

Interpretations concerning only a single isolated aetiological fraction also have to be considered. The definition of the aetiological fraction is made in the context of a hypothetical situation ("if the risk factor had not occurred"), so caution is needed in the interpretation of the estimate and in the translation into preventive potential. Even if the risk factor were completely removed at a given moment, some time may elapse before the incidence level among the previously exposed reaches the level among the never exposed. Nonetheless the fraction is informative. It provides information about the aetiology of the disease in the actual population as well as what can maximally be obtained by a change of habits in the next generation; but it does not indicate whether this change can easily be obtained.

If some of the assumed causal relations are not agreed upon due to lack of "final proofs" of causality, the calculated aetiological fractions are still of some interest. They can then be regarded as indicators of the possible size of impact on public health of the risk factor. A large aetiological fraction calls for further research to clarify whether the observed associations really are causal.

\section{BIAS}

It has to be stressed once again that the intrinsic interpretation of the aetiological fractions is crucially dependent on the assumed causal relationships. If these assumptions are agreed, the next question is whether the estimated aetiological fractions are biased in any direction.

We have tried to use conservative estimates of both the relative risks and the fractions of exposed individuals in the calculations in this paper. The remaining question is whether the assumptions made introduce any particular bias. The interactions between the studied risk factors have seldom been studied, but when this is done the interactions have been shown to be small. ${ }^{22} 23$ This is in accordance with the general experience that the multiplicative model is a good description of most data. ${ }^{15}$ Deviation from the assumption of no interaction probably introduces only minor bias.

Possible deviations from the assumption of independent distribution of risk factors can be in two directions. The risk factor is either more or less frequent in the presence of some other risk factors. An example of the first would be that shift work was more prevalent among workers with monotonous high paced work. An example of the latter would occur if "sedentary" workers were less exposed to noise than non-sedentary workers. When a multiplicative model is assumed and the risk factors are positively correlated, insertion of an adjusted relative risk into the simplistic formula leads to an underestimation. Similarly a negative correlation would lead to an overestimation. The first type of situation is probably the more common, but it has not been possible to calculate the correlation between the risk factors in Denmark. Consequently the bias in the estimate of aetiological fractions is not calculated either.

The most serious bias is probably due to the way in which the estimation of the number of people exposed has been performed and to the way in which the studied population has been delimited. The proportion of people exposed is the proportion of currently exposed people. But for some of the risk factors it might be more relevant to use the proportion of the population that has been exposed. Unfortunately these figures do not exist. Monotonous high paced work and shift work are probably more common among younger people. A selection and healthy worker effect like this biases cross sectional studies toward no association while cohort studies will still show an association. This is in fact the case in the studies of shift work. ${ }^{2}$ To what extent the increased risk is fading out and how fast it happens when people change from shift work to regular hours is not well described. We are not able to quantify the bias, but it might lead to considerable underestimation. We consider this selection bias to be negligible in relation to passive smoking and small in relation to noise.

We have been dealing with the working population and not the entire population. Applying the estimated aetiological fraction to the entire population would overestimate the impact 
of working conditions on cardiovascular health This bias would be larger among women, as a considerable proportion has not worked regularly outside the home, and minor among men, as almost all men are (or have been) working.

\section{CONCLUSION}

The calculations presented in this paper give a provisional impression of the quantitative impact of working conditions on cardiovascular diseases. The impact appears to be rather large. We feel that work environment factors ought to play a bigger role in cardiovascular epidemiological research in the future and also that the cardiovascular diseases should be acknowledged as work related diseases in the same way as cancer, reproductive failures, lung diseases, and many others. It is evident that more-and betterresearch is needed, but this fact ought not be used as an excuse to postpone preventive programmes aiming at improving the work environment.

The authors thank Lis Olsen for language revision and Professor Gavin Mooney for helpful comments on the paper. Supported by the Danish Work Environment Fund, the Danish Heart Foundation, and the University of Copenhagen. environment-a critical review of the and work literature on non-chemical factors. Health 1989; 15: 165-79.

2 Kristensen TS. Cardiovascular diseases and work environment-a critical review of the epidemiologic literature on chemical factors. Scand $\mathcal{f}$ Work Environ Health 1989; 15: 245-64.

3 Fox AJ, Adelstein AM. Occupational mortality: work or way of life? F Epidemiol Community Health 1978; 32: 73-8.

$4 \mathrm{Kannel}$ WB. Some lessons in cardiovascular epidemiology from Framingham. Am $\mathcal{f}$ Cardiol 1976; 37: 269-82.
5 Morkeberg H. Arbejdstidens placering. Meddelelse nr. 29. København: Socialforskningsinstituttet, 1980.

6 Olsen $\mathrm{O}$, Kristensen TS. Hjerte/karsygdomme og arbejdsmiljo. Bind 3. København: Arbejdsmiljøfondet, arbejdsm.

7 Paffenbarger RS, Hyde RT, Wing AL, et al. A natural history of athleticism and cardiovascular health. $\mathscr{f} A M A$ history of athlet

8 Morris JN, Everitt MG, Pollard R, et al. Vigorous exercise in leisure-time: Protection against coronary heart disease. Lancet 1980; ii: 1207-10

9 Groth MV. Fysisk aktivitet og motionsvaner $i$ den danske befolkning 1987. København: Dansk Institut for Klinisk Epidemiologi, 1988.

10 Levin ML. The ocurrence of lung cancer in man. Acta Unio Int Contra Cancrum 1953; 9: 531-41.

11 Miettinen OS. Proportion of disease caused or prevented by a given exposure, trait or intervention. $\mathrm{Am} \mathcal{F}$ Epidemio 1974; 99: 325-32.

12 Walter SD. Effects of interaction, confounding and observational error on attributable risk estimation. $A m \mathcal{F}$ Epidemiol 1983; 117: 598-604.

13 Greenland S, Robins JM. Conceptual problems in the definition and interpretation of attributable fractions. $A m \mathcal{F}$ Epidemiol 1988; 128: 1185-97.

14 Bruzzi P, Green SB, Byar DP, et al. Estimating the population attributable risk for multiple risk factors using case control data. Am $\mathcal{F}$ Epidemiol 1985; 122: 904-14.

15 Breslow NE, Day NE. Statistical methods in cancer research. Vol 1. The analysis of case-control studies. Lyon: IARC, 1980.

16 Rothman KJ. Modern epidemiology. Boston: Little, Brown, 1986.

17 Theorell T, Karasek R. Kan vi minska antalet hjärtinfarkter genom at förbättra den psykosociala arbetsmiljön? Läkartidningen 1989; 86: 1455-6.

18 Kawachi MB, Pearce NE, Jackson RT. Deaths from lung cancer and ischaemic heart disease due to passive smoking in New Zealand. NZ Med $\mathcal{f} 1989$; 102: 337-40.

19 Anonymous. Protective effect of physical activity on coronary heart disease. MMWR 1987; 36: 426-30

20 Wilhelmsen L. Kranskärlsjukdom og cerebrovaskulär sjukdom-Kunskapsunderlag till 1987 ärs folkhälso rapport. Stockholm: Socialstyrelsen 1987.

21 Paffenbarger RS, Hyde RT, Wing AL, Hsieh CC. Physical activity, all-cause mortality and longevity of college alumni (letter). N Engl f Med 1986; 315: 400-1.

22 Hattis D, Richardson B. Noise, general stress response, and cardiovascular disease processes: review and reassessment of hypothesized relationships. Massachusetts: MIT , 1980 .

23 Gotto AM. Interactions. Massachusetts: MIT, 1980. Gotto AM. Interactions of the major risk factors for 48-55.

\section{Commentary}

The effects of occupation on the incidence of cardiovascular disease have been seriously underreported in published work. Public health epidemiologists tend to concentrate almost exclusively on life style factors while occupational health professionals prefer to report the influence of work on the incidence of cancer, reproductive outcome, or musculoskeletal disorders. The imbalance is partly restored by this thought provoking paper of Olsen and Kristensen.

The standard texts in occupational medicine give little space to cardiovascular disease despite the fact that it is a major contributor to Western mortality and morbidity. Two recent and thorough reviews by, incidentally, one of the authors of the current paper, cite a number of chemical and non-chemical workplace factors which can (or might) influence cardiovascular disease. ${ }^{12}$ Of the chemical factors, carbon disulphide and nitroglycerine/nitroglycerol are well recognised causes, although recent studies suggest that carbon disulphide may act more through a reversible, direct cardiotoxic or thrombotic influence than through previously assumed atherogenic effect. ${ }^{3}$ Nitroglycerine can cause both acute and chronic effects and is well known for the disturbing "Monday morning death" syndrome. Some chemicals, such as methylene chloride, cause carboxyhaemoglobinaemia while other chlorinated hydrocarbons appear to cause cardiac arrhythmias. Inconclusive effects, possibly in some cases secondary to hypertension, have been ascribed to lead, cadmium, arsenic, and antimony. Recent reports suggest that epichlorohydrin ${ }^{5}$ and hydrogen sulphide ${ }^{6}$ might be added to the list. Peripheral vascular effects certainly arise from workplace exposure to cold, vibration and vinyl chloride monomer.

While chemical exposures are relatively rare, the influence of putative circumstances such as work "stressors", shift work, and sedentary occupations has received less attention but might be of greater preventive health importance. The difficulty has always been how to tease out these influences from life style when reviewing the mass of health data related to working populations. Olsen and Kristensen have attempted to do just that.

Using a scoring system for the quality of epidemiological studies, they calculated crude relative risks using quality in combination with quantity and consistency of findings in published reports. Combining this with a rough guide to the 